\title{
Hermite-Hadamard Type Inequalities for Composite Log-Convex Functions
}

\author{
Nik Muhammad Farhan Hakim Nik Badrul Alam,"*, Ajab Bai Akbarally², Silvestru Sever Dragomir ${ }^{3}$ \\ ${ }^{1}$ Faculty of Computer and Mathematical Sciences, Universiti Teknologi MARA Cawangan Pahang, Malaysia \\ ${ }^{2}$ Faculty of Computer and Mathematical Sciences, Universiti Teknologi MARA Shah Alam, Malaysia \\ ${ }^{3}$ Mathematics, College of Engineering and Science, Victoria University, Australia
}

Received February 19, 2020; Revised May 4, 2020; Accepted May 23, 2020

Copyright $@ 2020$ by authors, all rights reserved. Authors agree that this article remains permanently open access under the terms of the Creative Commons Attribution License 4.0 International License

\begin{abstract}
Hermite-Hadamard type inequalities related to convex functions are widely being studied in functional analysis. Researchers have refined the convex functions as quasi-convex, $h$-convex, log-convex, $m$-convex, $(\alpha, m)$-convex and many more. Subsequently, the Hermite-Hadamard type inequalities have been obtained for these refined convex functions. In this paper, we firstly review the Hermite-Hadamard type inequality for both convex functions and log-convex functions. Then, the definition of composite convex function and the Hermite-Hadamard type inequalities for composite convex functions are also reviewed. Motivated by these works, we then make some refinement to obtain the definition of composite log-convex functions, namely composite- $\varphi^{-1}$ log-convex function. Some examples related to this definition such as $G G$-convexity and $H G$-convexity are given. We also define $k$-composite log-convexity and $k$-composite- $\varphi^{-1}$ log-convexity. We then prove a lemma and obtain some Hermite-Hadamard type inequalities for composite log-convex functions. Two corollaries are also proved using the theorem obtained; the first one by applying the exponential function and the second one by applying the properties of $k$-composite log-convexity. Also, an application for $G G$-convex functions is given. In this application, we compare the inequalities obtained from this paper with the inequalities obtained in the previous studies. The inequalities can be applied in calculating geometric means in statistics and other fields.
\end{abstract}

Keywords Convex Functions, Hermite-Hadamard Inequalities, Composite Log-Convex Functions,

\section{Introduction}

Let $\psi: J \subseteq \mathbb{R} \rightarrow \mathbb{R}$ be a convex function on $J$ with $c, d \in J$ such that $c<d$. Then

$$
\psi\left(\frac{c+d}{2}\right) \leq \frac{1}{d-c} \int_{c}^{d} \psi(\tau) d \tau \leq \frac{\psi(c)+\psi(d)}{2}
$$

is known as the Hermite-Hadamard inequality.

Let $\varphi[c, d] \rightarrow[\varphi(c), \varphi(d)]$ be continuous, strictly increasing and differentiable on $(c, d)$.

Dragomir and Mond [1] refined the Hermite-Hadamard inequality in (1) for log-convex functions. The result obtained is as follows:

Let $\psi:[c, d] \rightarrow(0, \infty)$ be a log-convex function, then the inequality

$\ln \psi\left(\frac{c+d}{2}\right) \leq \frac{1}{d-c} \int_{C}^{d} \ln \psi(\tau) d \tau \leq \frac{\ln \psi(c)+\ln \psi(d)}{2}$

is known as an inequality of Hermite-Hadamard type for log-convex functions.

The definition of composite convex function was given by Dragomir [2]. The definition is stated below:

Definition 1. A function $\psi:[c, d] \rightarrow \mathbb{R}$ is composite $-\varphi^{-1}$ convex on the interval $[c, d]$ if the composite convex function $\psi \circ \varphi^{-1}:[\varphi(c), \varphi(d)] \rightarrow \mathbb{R}$ is convex on $[\varphi(c), \varphi(d)]$.

Let $\psi:[c, d] \rightarrow \mathbb{R}$ be a composite- $\varphi^{-1}$ convex function on $[c, d]$, then 
$\psi \circ \varphi^{-1}((1-\gamma) \alpha+\gamma \beta) \leq(1-\gamma) \psi \circ \varphi^{-1}(\alpha)+\gamma \psi \circ \varphi^{-1}(\beta)$

holds for any $\alpha, \beta \in[\varphi(c), \varphi(d)]$ and $\gamma \in[0,1]$. It can also be seen that this is equivalent to

$$
\psi \circ \varphi^{-1}((1-\gamma) \varphi(m)+\gamma \varphi(n)) \leq(1-\gamma) \psi(m)+\gamma \psi(n)
$$

for any $m, n \in[c, d]$ and $\gamma \in[0,1]$.

Dragomir [2] also introduced the concept of $k$-composite convexity by assuming that $\psi:[c, d] \rightarrow I$, where $I$ is a real number interval and $k: I \rightarrow \mathbb{R}$ is continuous and strictly increasing on $I$.

Definition 2. $\psi:[c, d] \rightarrow I$ is said to be k-composite convex on $[c, d]$ if the composite function $k \circ \psi$ is convex on $[c, d]$.

Since $\varphi:[c, d] \rightarrow[\varphi(c), \varphi(d)]$ is continuous, strictly increasing and differentiable on $(c, d)$, with $\psi:[c, d] \rightarrow I$, where $I$ is an interval of real numbers and $k: I \rightarrow \mathbb{R}$ is a continuous function on $I$ that is strictly increasing on $I$, Dragomir [2] also considered:

Definition 3. A function $\psi:[c, d] \rightarrow I$ is $k$-composite- $\varphi^{-1}$ convex on $[c, d]$, if $k \circ \psi \circ \varphi^{-1}$ is convex on $[\varphi(c), \varphi(d)]$.

Clearly, the above definition is equivalent to the condition

$$
\begin{aligned}
k \circ \psi \circ \varphi^{-1}((1-\gamma) \varphi(m)+\gamma \varphi(n)) \\
\quad \leq(1-\gamma)(k \circ \psi)(m)+\gamma(k \circ \psi)(n)
\end{aligned}
$$

for any $m, n \in[c, d]$ and $\gamma \in[0,1]$.

The Hermite-Hadamard inequality for composite- $\varphi^{-1}$ convex function has already been proved by Dragomir [2]. The result is given below.

Theorem 4. Let $\varphi:[c, d] \rightarrow[\varphi(c), \varphi(d)]$ be $a$ continuous strictly increasing and differentiable on $(c, d)$. If $\psi:[c, d] \rightarrow \mathbb{R}$ is composite $-\varphi^{-1}$ convex on $[c, d]$, then

$$
\begin{aligned}
& \psi \circ \varphi^{-1}\left(\frac{\varphi(c)+\varphi(d)}{2}\right) \\
& \leq \gamma \psi \circ \varphi^{-1}\left(\frac{(2-\gamma) \varphi(c)+\gamma \varphi(d)}{2}\right) \\
& \quad+(1-\gamma) \psi \circ \varphi^{-1}\left(\frac{(1-\gamma) \varphi(c)+(1+\gamma) \varphi(d)}{2}\right) \\
& \leq \frac{1}{\varphi(d)-\varphi(c)} \int_{C}^{d} \psi(\tau) \varphi^{\prime}(\tau) d \tau \\
& \leq \frac{1}{2}\left[\psi \circ \varphi^{-1}(1-\gamma) \varphi(c)+\gamma \varphi(d)+(1-\gamma) \psi(d)\right] \\
& \leq \frac{\psi(c)+\psi(d)}{2}
\end{aligned}
$$

for any $\gamma \in[0,1]$.

Dragomir [3] then refined the concept of composite convex function and obtained the definition of composite $h$-convex function. Furthermore, the Hermite-Hadamard inequalities for composite $h$-convex function were obtained.

Recently, Kashuri et al. [4] defined composite preinvex functions and $k$-composite preinvex functions. They obtained some Ostrowski inequalities for these functions.

\section{Composite Log-Convex Functions}

In this paper, we extend the definition above to define composite log-convex functions as follows.

Definition 5. A function $\psi:[c, d] \rightarrow(0, \infty)$ is said to be composite- $\varphi^{-1}$ log-convex on $[c, d]$ if $\psi \circ \varphi^{-1}:[\varphi(c), \varphi(d)] \rightarrow(0, \infty)$ is log convex on $[\varphi(c), \varphi(d)]$.

If $\psi:[c, d] \rightarrow(0, \infty)$ is composite- $\varphi^{-1}$ log-convex on

$[c, d]$, then we have

$$
\begin{aligned}
\ln \psi \circ \varphi^{-1}((1-\gamma) \alpha+\gamma \beta) \\
\quad \leq(1-\gamma)\left[\ln \psi \circ \varphi^{-1}(\alpha)\right]+\gamma\left[\ln \psi \circ \varphi^{-1}(\beta)\right] .
\end{aligned}
$$

On the other hand, the above inequality is equivalent to

$$
\begin{aligned}
& \psi \circ \varphi^{-1}((1-\gamma) \alpha+\gamma \beta) \\
& \quad \leq\left[\psi \circ \varphi^{-1}(\alpha)\right]^{1-\gamma}\left[\psi \circ \varphi^{-1}(\beta)\right]^{\gamma}
\end{aligned}
$$

for any $\alpha, \beta \in[\varphi(c), \varphi(d)]$ and $\gamma \in[0,1]$.

Note that the above inequality is also equivalent to

$$
\psi \circ \varphi^{-1}((1-\gamma) \varphi(m)+\gamma \varphi(n)) \leq[\psi(m)]^{1-\gamma}[\psi(n)]^{\gamma} .
$$

From Definition 5, suppose that $\varphi(n)=\ln n$, $n \in[c, d] \subset(0, \infty)$ then condition (9) becomes

$$
\psi\left(m^{1-\gamma} n^{\gamma}\right) \leq[\psi(m)]^{1-\gamma}[\psi(n)]^{\gamma}
$$

for any $m, n \in[c, d]$ and $\gamma \in[0,1]$, which is $G G$-convex as considered in [5].

By letting $\varphi(n)=-1 / n$, then condition (9) becomes

$$
\psi\left(\frac{n m}{(1-\gamma) n+\gamma m}\right) \leq[\psi(m)]^{1-\gamma}[\psi(n)]^{\gamma}
$$


for any $m, n \in[c, d]$ and $\gamma \in[0,1]$, which is $H G$-convex as considered in [5].

Next, we extend the concept of $k$-composite convexity from Definition 2 to define $k$-composite log-convexity as follows:

Definition 6. A function $\psi:[c, d] \rightarrow(0, \infty)$ is said to be $k$-composite log-convex on $[c, d]$, if $k \circ \ln \psi$ is convex on $[c, d]$.

Hence, the concept of $k$-composite- $\varphi^{-1}$ log-convexity is also introduced.

Definition 7. A function $\psi:[c, d] \rightarrow(0, \infty)$ is said to be $k$-composite- $\varphi^{-1}$ log-convex on $[c, d]$, if $k \circ \ln \psi \circ \varphi^{-1}$ is convex on $[\varphi(c), \varphi(d)]$.

The above definition can also be illustrated by the following inequality:

$$
\begin{aligned}
k \circ \ln \psi \circ \varphi^{-1}((1-\gamma) \varphi(m)+\gamma \varphi(n)) \\
\quad \leq(1-\gamma)(k \circ \ln \psi)(m)+\gamma(k \circ \ln \psi)(n)
\end{aligned}
$$

for any $m, n \in[c, d]$ and $\gamma \in[0,1]$.

Let $k$ be strictly increasing on $(0, \infty)$, then the inequality (12) is equivalent to

$$
\begin{aligned}
\ln \psi \circ \varphi^{-1}((1-\gamma) \varphi(m)+\gamma \varphi(n)) \\
\quad \leq k^{-1}[(1-\gamma)(k \circ \ln \psi)(m)+\gamma(k \circ \ln \psi)(n)]
\end{aligned}
$$

for any $m, n \in[c, d]$ and $\gamma \in[0,1]$.

\section{Some Refinements}

In this section, we obtain the Hermite-Hadamard inequalities for composite- $\varphi^{-1}$ log-convex function using the definitions which have been given in the previous section. Before the desired inequalities could be obtained, we state and prove the following lemma:

Lemma 8. Let $\psi:[c, d] \rightarrow(0, \infty)$ be a log-convex function on $[c, d]$. Then

$$
\begin{aligned}
& \ln \psi\left(\frac{c+d}{2}\right) \\
& \leq \gamma \ln \psi\left(\frac{(2-\gamma) c+\gamma d}{2}\right) \\
& \quad+(1-\gamma) \ln \psi\left(\frac{(1-\gamma) c+(1+\gamma) d}{2}\right) \\
& \leq \frac{1}{d-c} \int_{C}^{d} \ln \psi(\tau) d \tau \\
& \leq \frac{\gamma \ln \psi(c)+\ln \psi((1-\gamma) c+\gamma d)+(1-\gamma) \ln \psi(d)}{2} \\
& \leq \frac{\ln \psi(c)+\ln \psi(d)}{2}
\end{aligned}
$$

for any $\gamma \in[0,1]$.

Proof. Consider the Hermite-Hadamard inequality for log-convex function (2) and apply it on $[c,(1-\gamma) c+\gamma d]$, where $\gamma \in[0,1]$. Then, we obtain

$$
\begin{aligned}
& ((1-\gamma) c+\gamma d-c) \ln \psi\left(\frac{c+(1-\gamma) c+\gamma d}{2}\right) \\
& \leq \int_{C}^{(1-\gamma) c+\gamma d} \ln \psi(\tau) d \tau \\
& \leq((1-\gamma) c+\gamma d-c) \frac{\ln \psi(c)+\ln \psi((1-\gamma) c+\gamma d)}{2} .
\end{aligned}
$$

The above inequality is equivalent to

$$
\begin{aligned}
& \gamma \ln \psi\left(\frac{(2-\gamma) c+\gamma d}{2}\right) \\
& \leq \frac{1}{d-c} \int_{C}^{(1-\gamma) c+\gamma d} \ln \psi(\tau) d \tau \\
& \leq \frac{\gamma \ln \psi(c)+\gamma \ln \psi((1-\gamma) c+\gamma d)}{2} .
\end{aligned}
$$

We repeat the same process, but this time on interval $[(1-\gamma) c+\gamma d, d]$ with $\gamma \in[0,1]$ to obtain

$$
\begin{aligned}
& (1-\gamma) \ln \psi\left(\frac{(1-\gamma) c+(1+\gamma) d}{2}\right) \\
& \leq \frac{1}{d-c} \int_{(1-\gamma) c+\gamma d}^{d} \ln \psi(\tau) d \tau \\
& \leq \frac{(1-\gamma) \ln \psi((1-\gamma) c+\gamma d)+(1-\gamma) \ln \psi(d)}{2} .
\end{aligned}
$$

The second and third inequality in (14) are obtained by adding up (15) and (16). As the log-convexity property holds, then the following inequality is obtained:

$$
\begin{aligned}
& \gamma \ln \psi\left(\frac{(2-\gamma) c+\gamma d}{2}\right)+(1-\gamma) \ln \psi\left(\frac{(1-\gamma) c+(1+\gamma) d}{2}\right) \\
& \geq \ln \psi\left(\gamma\left(\frac{(2-\gamma) c+\gamma d}{2}\right)+(1-\gamma)\left(\frac{(1-\gamma) c+(1+\gamma) d}{2}\right)\right) \\
& \geq \ln \psi\left(\frac{c+d}{2}\right) .
\end{aligned}
$$

Thus, we have proved the first inequality in (14). The fourth inequality can be obtained by log-convexity property.

Theorem 9. Suppose that $\varphi:[c, d] \rightarrow[\varphi(c), \varphi(d)]$ is a continuous strictly increasing function and differentiable on $[c, d]$. Suppose also that $\psi:[c, d] \rightarrow(0, \infty)$ is composite- $\varphi^{-1} \log$-convex on $[c, d]$, then

$$
\begin{aligned}
& \ln \psi \circ \varphi^{-1}\left(\frac{\varphi(c)+\varphi(d)}{2}\right) \\
& \leq \gamma \ln \psi \circ \varphi^{-1}\left(\frac{(2-\gamma) \varphi(c)+\gamma \varphi(d)}{2}\right) \\
& \quad \quad+(1-\gamma) \ln \psi \circ \varphi^{-1}\left(\frac{(1-\gamma) \varphi(c)+(1+\gamma) \varphi(d)}{2}\right) \\
& \leq \frac{1}{\varphi(d)-\varphi(c)} \int_{\varphi(d)}^{\varphi(d)} \ln \psi(\tau) \varphi^{\prime}(\tau) d \tau \\
& \leq \frac{\gamma \ln \psi(c)+\ln \psi \circ \varphi^{-1}((1-\gamma) \varphi(c)+\gamma \varphi(d))+(1-\gamma) \ln \psi(d)}{2} \\
& \leq \frac{\ln \psi(c)+\ln \psi(d)}{2}
\end{aligned}
$$


for any $\gamma \in[0,1]$.

Proof. From inequality (14), we have for $\alpha, \beta \in[\varphi(c), \varphi(d)]$ and $\gamma \in[0,1]$ that

$$
\begin{aligned}
& \ln \psi \circ \varphi^{-1}\left(\frac{\alpha+\beta}{2}\right) \\
& \leq \gamma \ln \psi \circ \varphi^{-1}\left(\frac{(2-\gamma) \alpha+\gamma \beta}{2}\right) \\
& \quad+(1-\gamma) \ln \psi \circ \varphi^{-1}\left(\frac{(1-\gamma) \alpha+(1+\gamma) \beta}{2}\right) \\
& \leq \frac{1}{\beta-\alpha} \int_{\alpha}^{\beta} \ln \psi \circ \varphi^{-1}(\tau) d \tau \\
& \leq \frac{1}{2}\left[\gamma \ln \psi \circ \varphi^{-1}(\alpha)+\ln \psi \circ \varphi^{-1}((1-\gamma) \alpha+\gamma \beta)\right. \\
& \left.\quad+(1-\gamma) \ln \psi \circ \varphi^{-1}(\beta)\right] \\
& \leq \frac{\ln \psi \circ \varphi^{-1}(\alpha)+\ln \psi \circ \varphi^{-1}(\beta)}{2} .
\end{aligned}
$$

If we take $\alpha=\varphi(c)$ and $\beta=\varphi(d)$, then we get

$$
\ln \psi \circ \varphi^{-1}\left(\frac{\varphi(c)+\varphi(d)}{2}\right)
$$

$\leq \gamma \ln \psi \circ \varphi^{-1}\left(\frac{(2-\gamma) \varphi(c)+\gamma \varphi(d)}{2}\right)$

$$
+(1-\gamma) \ln \psi \circ \varphi^{-1}\left(\frac{(1-\gamma) \varphi(c)+(1+\gamma) \varphi(d)}{2}\right)
$$

$\leq \frac{1}{\varphi(d)-\varphi(c)} \int_{\varphi(c)}^{\varphi(d)} \ln \psi \circ \varphi^{-1}(\tau) d \tau$

$\leq \frac{\gamma \ln \psi(c)+\ln \psi \circ \varphi^{-1}((1-\gamma) \varphi(c)+\gamma \varphi(d))+(1-\gamma) \ln \psi(d)}{2}$

$\leq \frac{\ln \psi(c)+\ln \psi(d)}{2}$.

By using the change of variable $\varphi^{-1}(\tau)=u, u \in[c, d]$ we have $\tau=\varphi(u), d \tau=\varphi^{\prime}(u) d u$ and

$$
\int_{\varphi(c)}^{\varphi(d)} \ln \psi \circ \varphi^{-1}(\tau) d \tau=\int_{C}^{d} \ln \psi(u) \varphi^{\prime}(u) d u
$$

and by (19), we get the result as in (17).

Corollary 10. Let $\varphi:[c, d] \rightarrow[\varphi(c), \varphi(d)]$ be a continuous strictly increasing function that is differentiable on $(c, d)$. If $\psi:[c, d] \rightarrow(0, \infty)$ is composite- $\varphi^{-1} \log$-convex on $[c, d]$, then

$$
\begin{aligned}
& \psi \circ \varphi^{-1}\left(\frac{\varphi(c)+\varphi(d)}{2}\right) \\
& \leq \psi \circ \varphi^{-1}\left(\frac{(2-\gamma) \varphi(c)+\gamma \varphi(d)}{2}\right)^{\gamma} \times \\
& \psi \psi \varphi^{-1}\left(\frac{(1-\gamma) \varphi(c)+(1+\gamma) \varphi(d)}{2}\right)^{(1-\gamma)}
\end{aligned}
$$

$$
\begin{aligned}
& \leq \exp \left[\frac{1}{\varphi(d)-\varphi(c)} \int_{\varphi(c)}^{\varphi(d)} \ln \psi \circ \varphi^{-1}(\tau) d \tau\right] \\
& \leq \sqrt{\psi(c)^{\gamma} \psi(d)^{1-\gamma} \psi \circ \varphi^{-1}((1-\gamma) \varphi(c)+\gamma \varphi(d))} \\
& \leq \sqrt{\psi(c) \psi(d)}
\end{aligned}
$$

for any $\gamma \in[0,1]$.

This result is obvious by applying the exponential function to each side of the inequality in (17).

Corollary 11. Let $\varphi:[c, d] \rightarrow[\varphi(c), \varphi(d)]$ be $a$ continuous strictly increasing function and differentiable on $(c, d)$ and $k:(0, \infty) \rightarrow \mathbb{R}$ is a continuous function on $(0, \infty)$. If $\psi:[c, d] \rightarrow(0, \infty)$ is $k$-composite- $\varphi^{-1}$ log-convex on $[c, d]$, then

$$
\begin{aligned}
& \ln \psi \circ \varphi^{-1}\left(\frac{\varphi(c)+\varphi(d)}{2}\right) \\
& \leq k^{-1}\left[\gamma k \circ \ln \psi \circ \varphi^{-1}\left(\frac{(2-\gamma) \varphi(c)+\gamma \varphi(d)}{2}\right)\right. \\
& \left.+(1-\gamma) k \circ \ln \psi \circ \varphi^{-1}\left(\frac{(1-\gamma) \varphi(c)+(1+\gamma) \varphi(d)}{2}\right)\right] \\
& \leq k^{-1}\left[\frac{1}{\varphi(d)-\varphi(c)} \int_{\varphi(c)}^{\varphi(d)} k \circ \ln \psi(\tau) \varphi^{\prime}(\tau) d \tau\right] \\
& \leq k^{-1}\left[\frac { 1 } { 2 } \left[\gamma k \circ \ln \psi(c)+k \circ \ln \psi \circ \varphi^{-1}((1-\gamma) \varphi(c)+\gamma \varphi(d))\right.\right. \\
& +(1-\gamma) k \circ \ln \psi(d)]] \\
& \leq k^{-1}\left[\frac{k \circ \ln \psi(c)+k \circ \ln \psi(d)}{2}\right]
\end{aligned}
$$

for any $\gamma \in[0,1]$.

Proof. From (17), we have

$$
\begin{aligned}
& k \circ \ln \psi \circ \varphi^{-1}\left(\frac{\varphi(c)+\varphi(d)}{2}\right) \\
& \leq \gamma k \circ \ln \psi \circ \varphi^{-1}\left(\frac{(2-\gamma) \varphi(c)+\gamma \varphi(d)}{2}\right) \\
& \quad+(1-\gamma) k \circ \ln \psi \circ \varphi^{-1}\left(\frac{(1-\gamma) \varphi(c)+(1+\gamma) \varphi(d)}{2}\right) \\
& \leq \frac{1}{\varphi(d)-\varphi(c)} \int_{\varphi(c)}^{\varphi(d)} k \circ \ln \psi(\tau) \varphi^{\prime}(\tau) d \tau \\
& \leq \frac{1}{2}\left[\gamma k \circ \ln \psi(c)+k \circ \ln \psi \circ \varphi^{-1}((1-\gamma) \varphi(c)+\gamma \varphi(d))\right. \\
& \quad+(1-\gamma) k \circ \ln \psi(d)] \\
& \leq \frac{k \circ \ln \psi(c)+k \circ \ln \psi(d)}{2}
\end{aligned}
$$

for any $\gamma \in[0,1]$.

Taking $k^{-1}$ into (22), we get the desired result as in (21). 


\section{Application for GG-Convex Function}

If we take $\varphi(n)=\ln n, \quad n \in[c, d] \subset(0, \infty)$, then $\psi:[c, d] \rightarrow(0, \infty)$ is $G G$-convex on $[c, d]$ with $k:(0, \infty) \rightarrow \mathbb{R}, k(n)=\ln n$. By making use of Corollary 11 , we obtain

$$
\begin{aligned}
\psi(\sqrt{c d}) & \leq \psi^{\gamma}\left(\sqrt{c^{2-\gamma} d^{\gamma}}\right) \psi^{1-\gamma}\left(\sqrt{c^{1-\gamma} d^{1+\gamma}}\right) \\
& \leq \exp \left[\frac{1}{\ln d / c_{c}^{d}} \int_{c}^{\ln \psi(n)} \frac{}{n} d n\right] \\
& \leq \sqrt{\psi^{\gamma}(c) \psi^{1-\gamma}(d) \psi\left(c^{1-\gamma} d^{\gamma}\right)} \\
& \leq \sqrt{\psi(c) \psi(d)}
\end{aligned}
$$

for any $\gamma \in[0,1]$. This result can be compared to the inequalities obtained in [1], [6] and [7].

For further discussion, the result obtained in this section can also be compared to the inequalities of Hermite-Hadamard type related to $G G$-convex functions as obtained in [8-10].

\section{Conclusions}

The definition of composite convex function can be refined for some other functions, such as $h$-convex function, log-convex function and $(\alpha, m)$-convex function. Composite $h$-convex function was defined by Dragomir [3], composite log-convex function is defined in this paper and composite $(\alpha, m)$-convex function can be explored in future research.

The defined functions are used to refine the Hermite-Hadamard inequalities. The results obtained are related to $G G$-convexity, which can be applied in calculating the geometric means, especially in the field of statistics.

\section{Acknowledgements}

The first and second authors would like to thank Universiti Teknologi MARA (UiTM) for the support in the preparation and publication of this paper.

\section{REFERENCES}

[1] Dragomir, S. S., \& Mond, B. (1998). Integral inequalities of Hadamard type for log-convex functions. Demonstratio Mathematica, 31(2), 355-364.
[2] Dragomir, S. S. (2019). Inequalities of Hermite-Hadamard Type for Composite Convex Functions. In Frontiers in Functional Equations and Analytic Inequalities (pp. 559-584). Springer, Cham.

[3] Dragomir, S. S. (2018). Inequalities of Hermite-Hadamard Type for Composite $h$-Convex Functions. RGMIA Research Report Collection, Vol.21, Art.40.

[4] Kashuri, A., Liko, R. \& Iqbal, S. (2019). Fractional Integral Inequalities for Composite and $k$-Composite Preinvex Functions. Fasciculi Mathematici, 62, 57-79.

[5] Anderson, G. D., Vamanamurthy, M. K., \& Vuorinen, M. (2007). Generalized convexity and inequalities. Journal of Mathematical Analysis and Applications, 335(2), 1294-1308.

[6] Dragomir, S. S. (2018) Inequalities of Hermite-Hadamard Type for GG-Convex Functions. RGMIA Research Report Collection, Vol.18, Art.71.

[7] Dragomir, S. S. (2017). Some Integral Inequalities of Hermite-Hadamard Type For GG-Convex Functions. Mathematica, 59, 47-64.

[8] Dragomir, S. S. (2015). New Inequalities of Hermite-Hadamard Type for GG-Convex Functions. RGMIA Research Report Collection, Vol.18, Art.72.

[9] Dragomir, S. S. (2015). Some Integral Inequalities of Hermite-Hadamard Type for GG-Convex Functions. RGMIA Research Report Collection, Vol.18, Art.74.

[10] Dragomir, S. S. (2015). Weighted Integral Inequalities for GG-Convex Functions. RGMIA Research Report Collection, Vol.18, Art.75. 